\title{
Status Epilepticus, Hemispheric Confusion, a CT Perfusion Pitfall in "Code Stroke" Patients: A Case Report
}

\author{
Houman Sotoudeh ${ }^{1, *}$ \\ ${ }^{1}$ Department of Neuroradiology, University of Alabama in Birmingham (UAB), Birmingham, AL, United States \\ "Corresponding author: Department of Neuroradiology, University of Alabama in Birmingham (UAB), room 333, 619 19th St. S, Birmingham, AL 35294, United States. Email: \\ houmansotoudeh@gmail.com
}

Received 2018 January 28; Revised 2018 June 06; Accepted 2018 June 10.

\begin{abstract}
CT perfusion (CTP) is an evolving protocol for stroke evaluation in many hospitals, but there are several pitfalls in CT perfusion. Herein I present a patient suspicious of brain infarction under "stroke code". CT perfusion was initially visually interpreted and because of obvious asymmetry between the right and left cerebral hemispheres the interpretation was consistent with right cerebral ischemia. CT angiogram (CTA) did not demonstrate significant vascular stenosis. Subsequent electroencephalography (EEG) was consistent with status epilepticus from the left cerebral hemisphere. Since more and more CTPs are now performed in acute setting of code stroke more patients are being imaged with ongoing seizure with increased perfusion in epileptogenic brain parenchyma. This is different from the conventional concept of hypo perfusion of the involved brain in CTP performed in the "post-ictal" phase. The interpreting radiologist should be informed about the patient symptoms and should avoid solo visual assessment of CT perfusion in patients under the "stroke code".
\end{abstract}

Keywords: CT Perfusion, Stroke, Seizure, Status Epilepticus

\section{Introduction}

Brain ischemic stroke is a leading cause of death. The main principle of current stroke treatment approach is to start the treatment as soon as possible and to extend the window of treatment in cases with salvageable peri-infarct ischemia. This approach emphasizes the role of state of art imaging techniques to detect the ischemia and infarction with very high accuracy. In this context, CT perfusion is the main imaging modality given its availability. Many of these "code-stroke" CT perfusions are performed after hours and should be interoperated in minutes to reduce the time of initial treatment. The aforementioned facts make the role of the radiologist critical in stroke management. The radiologist should be familiar with many pitfalls of "codestroke" CT perfusion. There are several commercially available CTP software to help the interoperating radiologists, but they are prone to many pitfalls and can be misleading in some conditions. In this paper, I present one of the stroke-mimicker conditions with unusual findings in CT perfusion causing misleading software interpretation.

\section{Case Presentation}

The patient is a 57-year-old female with a history of chronic obstructive pulmonary disease (COPD), obesity, di- abetes mellitus, status post mechanical mitral valve replacement on coumadin $2.5 \mathrm{mg}$ daily with goal international normalized ratio (INR) 2.5 - 3.5 who was in rehabilitation facility for recovery after prolonged hospitalization for urospepsis and septic shock. She had an acute episode of loss of consciousness. The patient was found unresponsive. In physical exam, the pupils were dilated but responsive. There was mild left facial droop with right arm curled to the chest. The blood pressure was $183 / 109 \mathrm{mmHg}$. The blood sugar was $173 \mathrm{mg} / \mathrm{dL}$ and the oxygenation was normal. The Glasgow coma scale (GCS) was 8. Total national institutes of health stroke scale (NIHSS) was more than 10. The patient was intubated. Upon arrival to the emergency department stroke code was activated and non-contrast CT was performed which revealed no evidence of intracranial hemorrhage. There was no evidence for acute infarction on non-contrast CT (no evidence for dense middle cerebral artery [MCA] sign, insular ribbon sign or obscuration of lentiform nucleus). There was evidence of encephalomalacia and old cortical infarction in the left occipital lobe (Figure 1). As part of hospital stroke protocol, the patient underwent CT perfusion, which revealed obvious asymmetry between the right and left cerebral hemispheres with mean transit time (MTT) of the right cerebral hemisphere longer than the left side and the cerebral blood volume 
(CBV) and cerebral blood flow (CBF) of right hemisphere less than the left side. CT perfusion was read visually and with automatic software postprocessing as suspicious for extensive ischemia in the right cerebral hemisphere (Figures 2 and 3). The same time CT angiogram did not show evidence of flow limiting stenosis especially on the right side but mild asymmetry of cortical branches with right sided cortical branched smaller than on the left side (Figure 4). In conclusion, because of clinical suspicion of ischemia, CT angiogram (CTA) and CT perfusion (CTP) findings were concluded as likely vasospasm and ischemia in the right cerebral hemisphere. The patient did not receive stroke treatment or conventional angiogram. The patient later developed eye twitching, so electroencephalography(EEG) was requested that revealed continuous 2 - $3 \mathrm{~Hz}$ periodic epileptiform sharp periodic lateralized epileptiform discharges over the left hemisphere consistent with focal status epilepticus. The patient was admitted in ICU and was loaded with Keppra $3 \mathrm{~g}$ IV, then $2 \mathrm{~g}$ of fosphenytoin which was continued by Keppra 1500 mg twice daily (BID). Subsequently, facial asymmetry was resolved and neurologic examination of the upper and lower extremities was symmetric. The patient stayed intubated in the ICU because of respiratory distress. Upon reviewing the CT perfusion and CT angiogram, the asymmetry between right and left cerebral hemispheres was consistent with hyper perfusion of the left cerebral hemisphere rather than hypo perfusion of the right side which was evident based on the CT perfusion "absolute values" and the "color scale" (decreased MTT and increased CBV and CBF of the left hemisphere).

\section{Discussion}

Brain ischemic stroke is a leading cause of death that is caused by occlusion of the brain arteries. Nowadays the main focus of stroke treatment is on reducing the time of treatment and differentiation between the "core infarction" and "pre-infarction ischemia penumbra" because patients with a large area of peri-fraction ischemia benefit most from the thrombolysis and intra-arterial thrombectomy (1). Application of CT perfusion has increased in the recent years for evaluation of acute ischemia and differentiation between the core infarction and peri- infarction ischemia, but its application is not standard of care because of controversies about standard protocol and its effect on patients' prognosis. The main physiologic parameters evaluated by CT perfusion are CBF, CBV and MTT. Areas with markedly decreased $\mathrm{CBV} / \mathrm{CBF}$ are considered as infarction and the area with increased MTT and relatively preserved CBV or even slightly increased CBV is considered as "peri-infarction ischemic penumbra". The patients with large pre-infarction ischemia can benefit from thrombolysis and intra-arterial thrombectomy. Generally, patients with relatively small ischemia penumbra do not ben- efit from thrombolysis/thrombectomy and may just suffer from the treatment side effects.

The main principle of CT perfusion is obtaining multiple CT images during injection of the iodine contrast bolus. The increased parenchymal density is proportional to the iodine contrast and arterial blood flow. Generally, 35 to 50 milliliter of iodine contrast is injected via the antecubital vein followed by 20 cc of saline. CT acquisition starts five to seven seconds after contrast injection and it continues up to 75 to 90 seconds. The brain coverage CT perfusion depends on the scanner but most of the time the brain at the level of the basal ganglia is evaluated although the new scanners can cover the main part of the brain with a large field of view. Parameters of CBF, CBV, MTT and time to maximum (T-Max) maps are calculated mathematically by non-convolution or deconvolution techniques. Mainly, $\mathrm{CBV} / \mathrm{CBF}$ is used to for differentiation between core infarction versus peri-infarction ischemia. The area with decreased CBV/CBF is considered to be infarction and the area with increased MTT but relatively preserved CBV or even slightly increased CBV is considered as peri-infarction ischemia (2). The normal value of the mentioned CTP parameters can be different given different scanners and protocols. The stroke centers are advised to set their own normal values. Generally, MTT about 4 s, CBF about $60 \mathrm{~mL} / 100$ $\mathrm{g} / \mathrm{min}$ and $\mathrm{CBV}$ about $4 \mathrm{~mL} / 100 \mathrm{~g}$ are considered as normal for gray matter and MTT of $4.8 \mathrm{~s}$, CBF of $25 \mathrm{~mL} / 100 \mathrm{~g} / \mathrm{min}$ and CBV of $2 \mathrm{~mL} / 100 \mathrm{~g}$ are considered normal for white matter. In gray matter, CBF less than $10-25 \mathrm{~mL} / 100 \mathrm{~g} / \mathrm{min}$ and CBV less than $2 \mathrm{~mL} / 100 \mathrm{~g}$ are considered as core infarction and relative MTT increase $>145 \%$ but relatively preserved $\mathrm{CBV} / \mathrm{CBF}$ is considered as "ischemic penumbra" (3, 4).

Up to $30 \%$ of clinically diagnosed acute ischemic strokes are not true stroke but stroke mimics. There are several diseases that can mimic stroke (5). Stroke mimics can occur in up to $19 \%$ of patients initially treated for stroke and in up to $16.7 \%$ of patients receiving thrombolytic therapy. Seizures are among the most common stroke differential diagnosis because the ictal and postictal symptoms can mimic stroke symptoms. Focal neurologic deficits can be seen during and after seizure episodes. It can be difficult to distinguish transient, post-ictal neurological deficits following seizure from those because of cerebral ischemia (6). In the presented case, loss of consciousness and mild facial asymmetry of the left face (likely Todd's paralysis) was considered as stroke signs in initial clinical evaluation. It should be noted that loss of consciousness is not a common presentation of focal status epilepticus, but the EEG was localized to the left cerebral hemisphere in this case. During the seizure, the perfusion increases in the involved brain parenchyma but perfusion rapidly reduces, so generally, on CT perfusion there is decreased perfusion in the epileptogenic foci $(5,7,8)$. In one prior published study, 


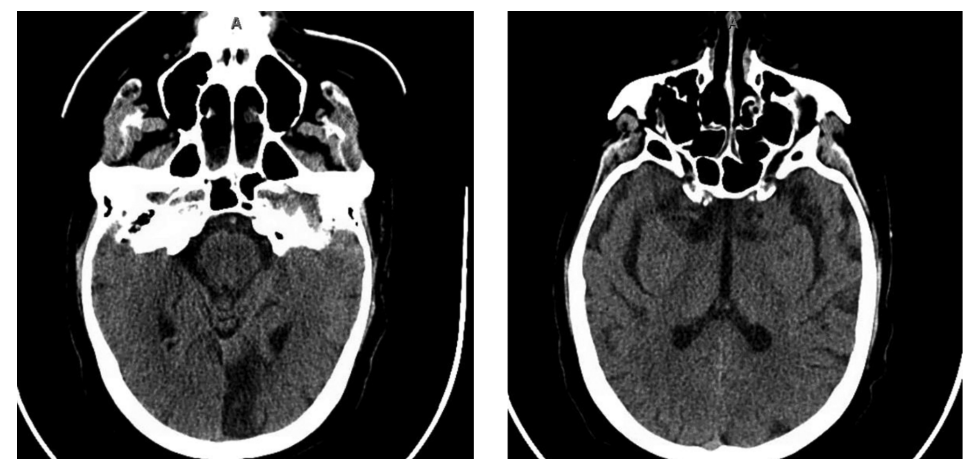

Figure 1. Non-contrast brain CT shows evidence of encephalomalacia and old infarction of the left occipital lobe without evidence of intracranial hemorrhage or obvious evidence of acute infarction such as dense middle cerebral artery (MCA) sign, ribbon insular sign and obscuration of the basal ganglia.
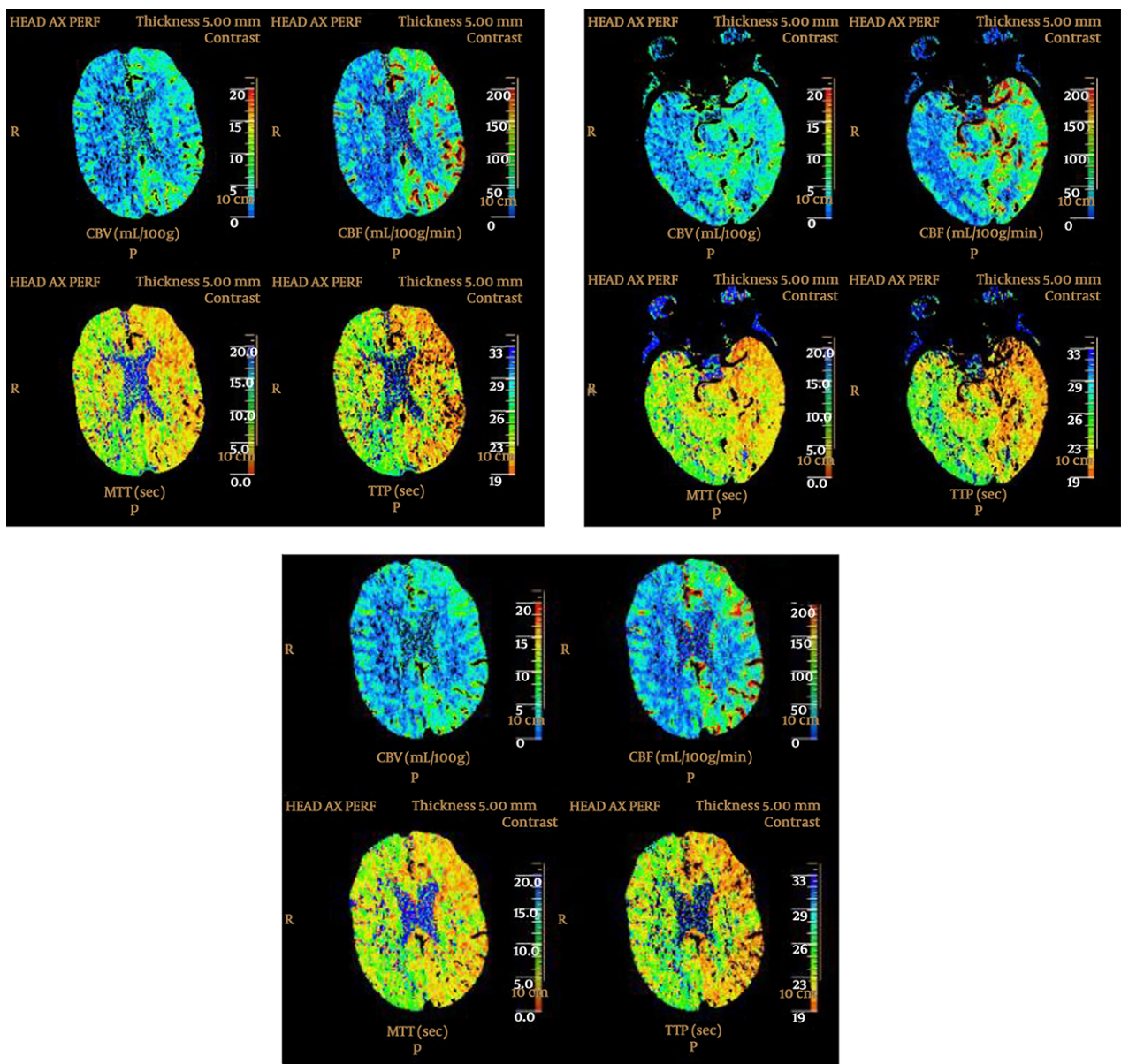

Figure 2. Evidence of obvious asymmetry between the cerebral hemispheres with longer mean transit time (MTT) and lower cerebral blood volume (CBV) and CBV in the right cerebral hemisphere. The CT perfusion was interpreted "visually". This pattern was reported as concerning for right cerebral ischemia but by looking at "color scale", the MTT of the left hemisphere is shorter than normal and the left sided CBV and CBV are more than normal (CBF, cerebral blood flow; TTP, time to peak ).

patients with epilepsy (and without infarction) were evaluated by CT perfusion up to 72 hours after the seizure at- tack. Thirty seven percent of these patients had abnormal perfusion with focal hypo perfusion with prolonged MTT 

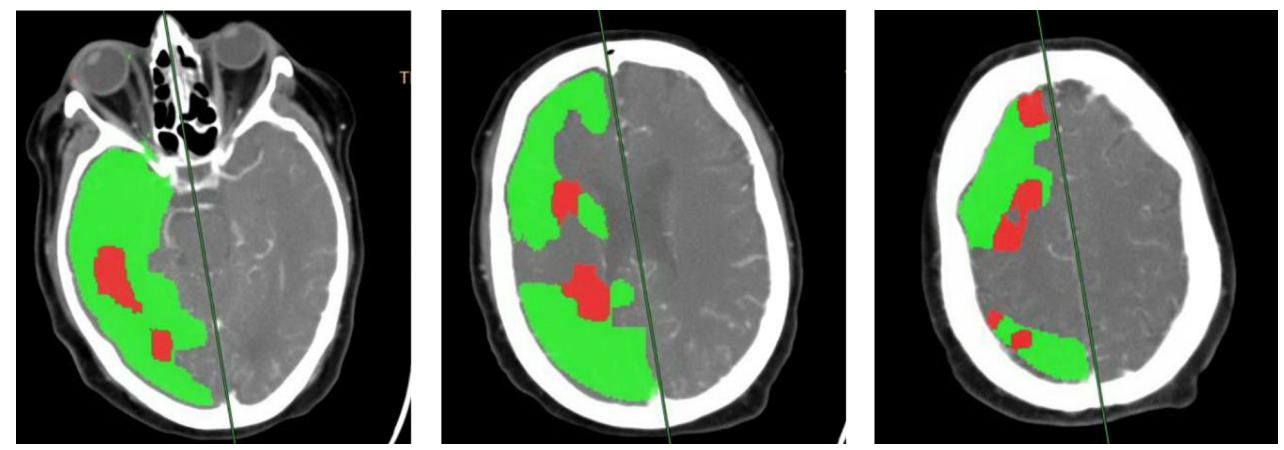

Figure 3. Prediction color maps from the CT perfusion were processed automatically. The green color shows area of increased mean transit time (MTT) and preserved cerebral blood volume (CBV) which was diagnosed as ischemia by the software and the red color is the area with decreased CBV which was diagnosed as infarction by the software.

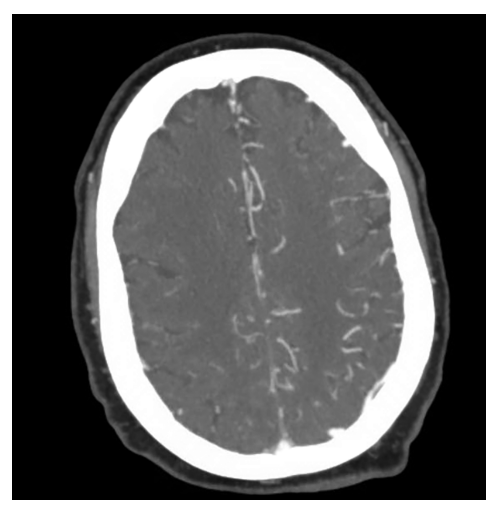

Figure 4. CT angiogram. Asymmetry between cortical branches of the right and left cerebral hemispheres which was first interpreted as suspicious for vasospasm of the right cerebral hemisphere.
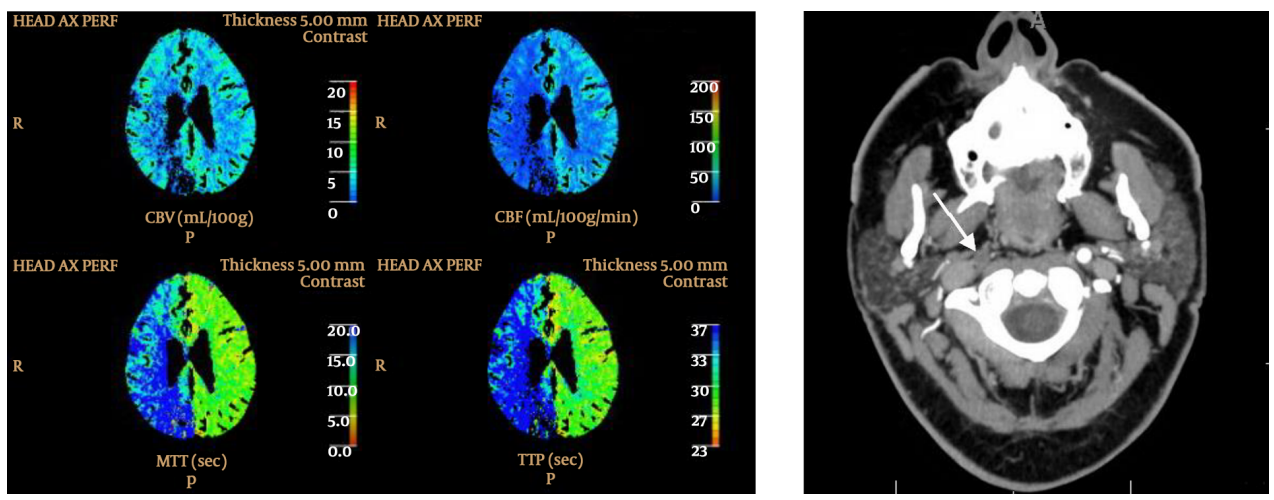

Figure 5. A companion case. CT perfusion in a patient under stroke code shows evidence of increased mean transit time (MTT) and decreased cerebral blood volume/cerebral blood flow (CBV/CBF) in the right cerebral hemisphere. This finding is real because the MTT, CBV and CBF of the left hemisphere are within normal limits. Subsequent CT angiogram showed complete occlusion of the right internal carotid artery (arrow) (TTP, time to peak).

and decreased $\mathrm{CBV}$ and $\mathrm{CBF}$ being the most common finding followed by hollo-hemispheric hypo perfusion, multilobar hypo perfusion and 'cortical ribbon' pattern (6). In our case, CTP was performed as stroke code few minutes after symptom presentation. CTP showed asymmetry in the right and left cerebral hemisphere with longer MTT and lower $\mathrm{CBF}$ and $\mathrm{CBV}$ in the right cerebral hemisphere in comparison to the left side. This asymmetry was because 
of left hollo-hemispheric hyperperfusion which was misinterpreted visually as right hollo-hemispheric hypoperfusion. The concurrent CT angiogram showed asymmetry of cortical branches of the right and left MCA arteries but without evidence of flow limiting stenosis in right-sided arterial circulation.

Figure 5 presents a companion case with similar holohemispheric asymmetry in CTP parameters which is identical to the aforementioned case. Visual assessment and the CTP software are not capable of differentiation between these two completely different pathologies. However, by measuring the absolute values of CTP parameters, the differentiation is possible. In the presented patient with seizure, the holo-hemispheric asymmetry is because of left hemispheric hyper perfusion so MTT and time to peak (TTP) of the left hemisphere are less than normal value and the $\mathrm{CBV}$ and $\mathrm{CBF}$ are more than the normal value. In the companion case, however, the holo-hemispheric asymmetry is because of right hemispheric hypo perfusion. So, MTT, TTP, CBV and CBF parameters of the left hemisphere are within normal limits (Figure 5).

Prior studies about CTP findings of seizure have been mostly performed post-ictally. Since CTP is now performed in acute setting as a part of stroke code, more CTPs are done during seizure and hyperperfusion of epileptogenic foci is more common than hypoperfusion. In one recent CTP study in "code stroke" patients, about $60 \%$ of seizure patients with stroke like symptoms showed hyperperfusion in the involved hemisphere (5). The radiologist should be aware of increased perfusion in epileptogenic foci and not interpret the contralateral hemisphere as ischemic. Any CTP for "code stroke "should be read by measuring the absolute CBV, CBF and MTT values. Being familiar with the normal perfusion value and paying attention to absolute $\mathrm{CBV}, \mathrm{CBF}$ and MTT values rather than solo visual assessment can help to avoid this confusion. In addition, the referring physician must provide information to the radiologist about any unusual symptoms that are not classical for ischemia and localize the suspected ischemic hemisphere that would help the radiologist to avoid confusion be- tween normal/hypoperfused hemisphere (ischemia) and normal/hyperperfused hemisphere (seizure).

\section{Footnotes}

Authors' Contributions: None declared.

Financial Disclosure: None declared.

Funding/Support: None declared.

\section{References}

1. Parsons MW, Pepper EM, Bateman GA, Wang Y, Levi CR. Identification of the penumbra and infarct core on hyperacute noncontrast and perfusion CT. Neurology. 2007;68(10):730-6. doi: 10.1212/01.wnl.0000256366.86353.ff. [PubMed: 17339580].

2. Heit JJ, Wintermark M. Perfusion Computed Tomography for the Evaluation of Acute Ischemic Stroke: Strengths and Pitfalls. Stroke. 2016;47(4):1153-8. doi: 10.1161/STROKEAHA.116.011873. [PubMed: 26965849].

3. Wintermark M, Flanders AE, Velthuis B, Meuli R, van Leeuwen M, Goldsher D, et al. Perfusion-CT assessment of infarct core and penumbra: receiver operating characteristic curve analysis in 130 patients suspected of acute hemispheric stroke. Stroke. 2006;37(4):979-85. doi: 10.1161/01.STR.0000209238.61459.39. [PubMed: 16514093].

4. Murphy BD, Fox AJ, Lee DH, Sahlas DJ, Black SE, Hogan MJ, et al. Identification of penumbra and infarct in acute ischemic stroke using computed tomography perfusion-derived blood flow and blood volume measurements. Stroke. 2006;37(7):1771-7. doi: 10.1161/01.STR.0000227243.96808.53. [PubMed: 16763182].

5. Austein F, Huhndorf M, Meyne J, Laufs H, Jansen O, Lindner T. Advanced CT for diagnosis of seizure-related stroke mimics. Eur Radiol. 2018;28(5):1791-800. doi: 10.1007/s00330-017-5174-4. [PubMed: 29218615].

6. Gelfand JM, Wintermark M, Josephson SA. Cerebral perfusion-CT patterns following seizure. Eur J Neurol. 2010;17(4):594-601. doi: 10.1111/j.1468-1331.2009.02869.x. [PubMed: 19968701].

7. O'Brien TJ, So EL, Mullan BP, Hauser MF, Brinkmann BH, Jack CR Jr, et al. Subtraction SPECT co-registered to MRI improves postictal SPECT localization of seizure foci. Neurology. 1999;52(1):137-46. doi: 10.1212/WNL.52.1.137. [PubMed: 9921861].

8. Wu RH, Bruening R, Noachtar S, Arnold S, Berchtenbreiter C, Bartenstein $P$, et al. MR measurement of regional relative cerebral blood volume in epilepsy. J Magn Reson Imaging. 1999;9(3):43540. doi: 10.1002/(SICI)1522-2586(199903)9:3<435::AID-JMRI11>3.0.CO;2J. [PubMed: 10194714]. 